\title{
Durée de jachère et valeur pastorale de la végétation herbacée en zone soudanienne au Sénégal
}

\author{
L.E. Akpo ${ }^{1}$ D. Masse ${ }^{2}$ M. Grouzis ${ }^{3}$
}

Mots-clés

Bétail - Parcours - Terre de pâturage Plante fourragère - Jachère - Qualité Sénégal.

\begin{abstract}
Résumé
La valeur pastorale des parcelles de jachères soudaniennes a été établie dans le terroir de Saré Yéro Bana, en Haute Casamance, en étudiant le spectre fourrager et l'indice global de qualité des terres de parcours. Une plus grande diversité $d^{\prime}$ espèces fourragères est apparue dans les parcours de jachères récentes. Dans les parcelles âgées, en revanche, les espèces de faible valeur pastorale ont été relativement plus abondantes. L'indice global de qualité des parcours a augmenté sensiblement au cours des premières années de l'abandon cultural, a culminé la troisième année en raison de l'abondance des graminées et du faible couvert ligneux, puis a diminué entre 5 et 17 ans. Cette baisse de la qualité fourragère des parcours de jachères a été encore plus accentuée lorsque la valeur pastorale nette a été évaluée. La prise en compte de la pondération de fourrage "qualifié » a amélioré la charge en bétail des jeunes jachères, car la part de fourrage inutilisée a pu être réduite de 50 p. 100. L'indice de qualité peut donc constituer un indicateur essentiel de la qualité fourragère des parcours, en I'absence de toute analyse chimique et/ou bromatologique.
\end{abstract}

\section{INTRODUCTION}

L'accroissement de la population et la nécessité de satisfaire des besoins alimentaires et domestiques qui deviennent de jour en jour plus importants conduisent à l'extension des surfaces cultivées. Les zones, même marginales, auparavant réservées au cheptel, sont désormais incluses dans le cycle culture-jachère. La jachère est l'état de la terre d'une parcelle entre la récolte d'une culture et le moment de l'introduction de la culture suivante. Elle joue divers rôles comme celui d'aider à la restauration de la fertilité des sols

1. Ucad/Fst/département de Biologie végétale, faculté des Sciences et techniques, BP 5005, Dakar, Sénégal

Tél. : (221) 6436518 ; fax : (221) 8242103 ; e-mail : akpo@dakar.ird.sn

2. Laboratoire Ecologie végétale, centre IRD, BP 1386, Dakar, Sénégal

E-mail : masse@ird.bf

3. Mission IRD, route d'Ambohipo, BP 434, Antananarivo 101, Madagascar

E-mail : grouzis@ird.mg
$(13,14)$, le contrôle des adventices des cultures, la diminution des risques d'érosion, l'amélioration de l'infiltration. Elle sert aussi de réserve foncière et peut constituer une source de bois de chauffe, de service, d'œuvre, de plantes médicinales, de petits fruits et d'usages divers. La jachère peut constituer aussi une réserve de pâturage pour les animaux (bovins, ovins et caprins) locaux ou transhumants (19). La végétation de jachère, en tant que parcours, comprend en effet entre 25 à 50 p. 100 d'espèces fourragères $(2,16,18,20,26)$. Connaître et mieux utiliser ce type de pâturage peut contribuer à diversifier les sources d'alimentation du bétail et, ainsi à réduire le temps d'utilisation des terres habituelles de parcours qui sont souvent dévastées par les feux de brousse et/ou les incendies de forêt, ou qui deviennent rapidement de qualité médiocre.

La qualité fourragère des jachères d'une zone soudanienne du sud du Sénégal a été analysée à partir de l'étude détaillée de la végétation en fonction du nombre d'années de repos cultural. L'étude a permis d'évaluer la quantité de fourrage disponible et de déduire le nombre d'animaux que l'on peut alimenter sur ce type de pâture. 


\section{MATERIEL ET METHODES}

\section{Zone de l'étude}

L'étude a été menée en Haute Casamance, dans la partie méridionale du Sénégal (figure 1), soit entre $12^{\circ} 50^{\prime}$ et $13^{\circ} 05^{\prime}$ de lat. $\mathrm{N}$ et entre $14^{\circ} 38^{\prime}$ et $14^{\circ} 58^{\prime}$ de long. $\mathrm{O}$. La région est caractérisée par une succession de bas plateaux au modelé plat et peu marqué. Le climat est tropical subhumide avec une longue saison sèche (sept mois) et une courte période humide (cinq mois). Les précipitations varient de 900 à $1100 \mathrm{~mm}$ par an, dont 60 à 80 p. 100 durant les mois d'août et de septembre. Les températures extrêmes moyennes mensuelles sont de $23{ }^{\circ} \mathrm{C}$ en janvier pour les plus faibles et de $32{ }^{\circ} \mathrm{C}$ en mai pour les plus élevées. La température moyenne annuelle est de $27^{\circ} \mathrm{C}$.

La végétation naturelle s'organise selon une toposéquence typique où les plateaux et les vallées alternent dans un continuum spatial à faibles contrastes (5). La végétation ligneuse haute des plateaux est dominée par Bombax costatum Pellegr. et Vuill., Pterocarpus erinaceus Poir., Daniellia oliveri (Rolfe) Hutch. et Dalz., Cordyla pinnata (Lepr. ex A. Rich.) Milne-Redh., Parkia biglobosa (Jacq.) Benth., Terminalia macroptera Guill. et Perr., tandis que la strate arbustive est largement dominée par Oxythenantera abyssinica (A. Rich.) Munro et Prosopis africana (Guill. et Perr.) Taub. Dans les vallées, l'emprise de l'agriculture est très forte et les arbres présents sont isolés. On y rencontre principalement Adansonia digitata L., Ceiba pentandra (L.) Gaertn., Ficus sp., Mitragyna inermis (Willd.) O. Ktze et Elaeis guineensis Jacq.

Le peuplement humain est constitué par les Peuhls fuladu qui pratiquent une agriculture diversifiée (céréales, arachide) étroitement associée à un élevage extensif sédentaire, créant des trouées de cultures et de jachères dans une vaste forêt claire sur glacis. Dans le système agraire fuladu, la jachère est au centre des relations entre l'agriculture et l'élevage. La jachère fournit du pâturage pour le bétail, le bois de feu et/ou des plantes médicinales pour la population.

\section{Méthodes}

L'étude de la végétation herbacée a été réalisée par une approche synchrone, consistant à choisir en même temps des parcelles de jachère d'âges différents. L'âge de la jachère a été déterminé par enquête (date de l'abandon cultural) auprès des propriétaires des parcelles. Cinq parcelles de jachères âgées de 1, 3, 5, 10 et 17 ans ont ainsi été retenues dans le terroir de Saré Yéro Bana en Haute Casamance, dans une région encore relativement épargnée par la demande foncière. Il a été supposé que les différences étaient seulement dues à l'âge des jachères et que les facteurs environnementaux étaient comparables. Ce terroir représente ainsi une sorte d'état initial non dégradé d'un système d'exploitation rural soudanien appelé à évoluer dans les années à venir, avec l'installation amorcée de plusieurs villages maraboutiques issus du bassin arachidier (12). L'abandon cultural intervient après trois ou quatre cycles de culture arachide-mil (ou maïs), c'est-à-dire après six ou huit années successives de culture.

La détermination de la valeur pastorale (VP) des herbages consiste à donner aux parcours un indice global de qualité (10) qui tient compte à la fois de la composition spécifique et de l'indice de qualité spécifique $\left(\mathrm{Is}_{\mathrm{i}}\right)$. La composition spécifique a été établie à partir des relevés de végétation dans les différentes jachères au maximum de végétation. Chaque relevé a été réalisé sur une superficie de $150 \mathrm{~m}^{2}$. L'échantillonnage a été constitué de 82 relevés pour l'ensemble du travail réparti entre les différents âges des parcelles de jachère retenus ( $\mathrm{J} 1=1$ an, 10 relevés ; $\mathrm{J} 3=3$ ans, 22 relevés ; $\mathrm{J} 5=5$ ans, 12 relevés ; $\mathrm{J} 10=10$ ans, 20 relevés ; J17 $=17$ ans, 18 relevés) au niveau du terroir de Saré Yéro Bana. Le nombre de relevés est apparu nettement plus important dans les parcelles de jachère de 3,10 et 17 ans en raison de l'hétérogénéité apparente de ces milieux. Les espèces ont été déterminées à l'aide de la Flore du Sénégal (4) et les synonymes actualisés sur la base de l'énumération des plantes à fleurs d'Afrique (21).

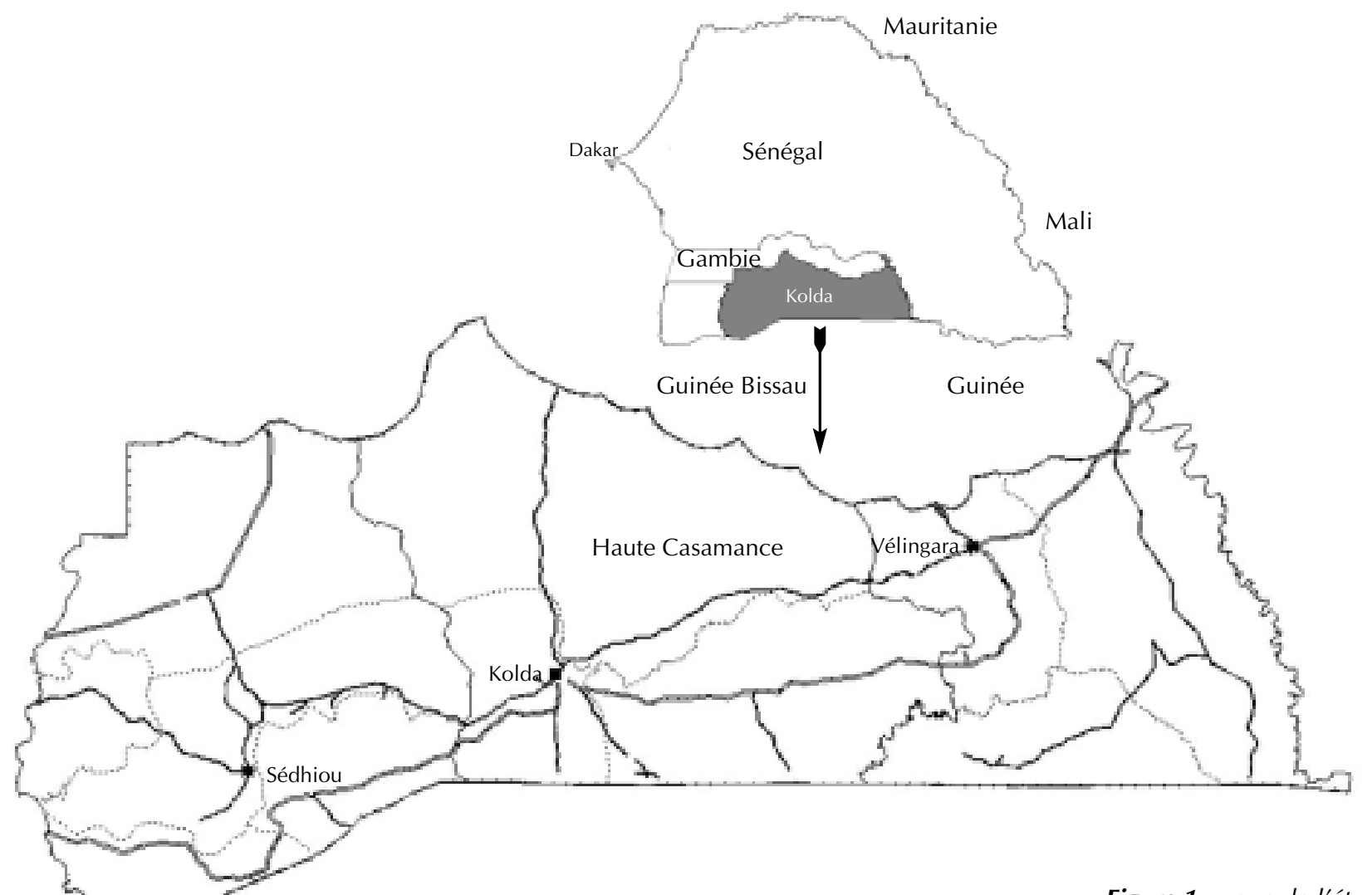

Figure 1 : zone de l'étude. 
L'indice spécifique traduit l'intérêt zootechnique, c'est-à-dire la valeur bromatolgique de chaque espèce végétale. La détermination repose sur l'appétibilité (ou l'appétence), la productivité (kg MS/ha/jour) et la valeur nutritive des espèces obtenue à partir de la composition chimique $(11,27)$. Ce critère de qualité, pour les espèces herbacées des terres de parcours de la zone sahélienne, est établi sur une échelle de cotation de 0 à $3(3,25)$, c'est-à-dire sur une échelle de quatre classes $(0,1,2$ et 3$)$ de la manière suivante :

- les plantes de bonne valeur pastorale (BVP) sont celles dont l'Is est égal à 3 ;

- les plantes de moyenne valeur pastorale (MVP) sont celles dont l'Is $s_{i}$ est égal à 2 ;

- les plantes de faible valeur pastorale (FVP) sont celles dont l'Is est égal à 1 ;

- les plantes sans valeur pastorale (SVP) sont celles dont l'Is $s_{i}$ est égal à 0 .

Ces différents groupes d'espèces (BVP, MVP, FVP et SVP) ou classes d'indices constituent dans la suite du texte les catégories d'espèces fourragères. La valeur pastorale est un indice synthétique qui prend aussi en compte la contribution spécifique $\left(\mathrm{Cs}_{\mathrm{i}}\right)$ au tapis herbacé.

La valeur pastorale a été calculée en multipliant les contributions des espèces $\left(\mathrm{Cs}_{\mathrm{i}}\right)$ par les indices de qualité correspondants $\left(\mathrm{Is}_{\mathrm{i}}\right)$; les valeurs pastorales relatives (VPr) des espèces ainsi obtenues sont additionnées puis divisées par le nombre de classes significatives d'indices $\left(\mathrm{Is}_{\mathrm{i}} \neq 0\right)$ et exprimées sur $100(10)$ :

$$
\mathrm{VP}=\frac{1}{3} \Sigma \mathrm{Cs}_{\mathrm{i}} \times \mathrm{Is}_{\mathrm{i}}
$$

La valeur ainsi obtenue est aussi appelée indice global ou indice synthétique de qualité. Celle-ci renseigne sur l'importance de fourrage « qualifié » (10) produit dans l'unité de milieu considéré. Lorsque l'on pondère en effet la production récoltée par la valeur de cet indice synthétique de qualité pastorale, on obtient la quantité de fourrage qualifié ; elle est exprimée de la façon suivante :

$$
\mathrm{Q}_{(\mathrm{kg} / \mathrm{ha})}=\mathrm{P}_{(\mathrm{kg} / \mathrm{ha})} \text { x VP }
$$

La production herbagère $\mathrm{P}(\mathrm{kg} \mathrm{MS} / \mathrm{ha})$ est évaluée au maximum de végétation par la méthode de récolte intégrale qui est considérée comme très acceptable pour estimer la productivité des communautés végétales à cycle court (28). La méthode consiste à couper la strate herbacée au ras du sol dans des placeaux d'un mètre carré. $\mathrm{Au}$ total 82 placeaux répartis entre les différents âges de jachère ont été utilisés. La capacité de charge est alors calculée sur la base du fourrage qualifié produit et pour une utilisation essentiellement de saison sèche de sept mois (novembre à mai).

\section{RESULTATS}

\section{Analyse de la composition botanique : le spectre fourrager}

La flore recensée dans les différentes parcelles de jachère a été riche de 60 espèces herbacées, réparties parmi 36 genres et 14 familles (tableau I). L'importance des genres et des espèces a été variable dans les différentes familles, comme suit :

Fabaceae : 8 genres, 16 espèces

Poaceae : 9 genres, 12 espèces

Convolvulaceae : 3 genres, 7 espèces

Euphorbiaceae : 3 genres, 3 espèces

Rubiaceae : 2 genres, 4 espèces

Amaranthaceae : 2 genres, 3 espèces

Asteraceae : 2 genres, 3 espèces
Caesalpiniaceae : 2 genres, 3 espèces

Cyperaceae : 2 genres, 3 espèces

Malvaceae : 2 genres, 3 espèces

Araceae : 1 genre, 1 espèce

Pedaliaceae : 1 genre, 1 espèce

Sterculiaceae : 1 genre, 1 espèce

Tiliaceae : 1 genre, 1 espèce

La famille des Fabaceae a été de loin la plus représentée, suivie de celles des Poaceae, des Convolvulaceae et des Rubiaceae. Ces trois premières familles ont ainsi rassemblé 35 espèces, soit 58,3 p. 100 de l'effectif total. Six autres familles (Amaranthaceae, Asteraceae, Caesalpiniaceae, Cyperaceae, Euphorbiaceae, Malvaceae) n'ont représenté que 30 p. 100 du total avec chacune d'elles trois espèces. Les autres familles (Araceae, Pedaliaceae, Sterculiaceae et Tiliaceae) n'ont été représentées que par une seule espèce. La super-famille des Leguminoseae (Fabaceae et Caesalpiniaceae) a rassemblé ainsi à elle seule près du tiers des espèces (19 espèces, soit 31,8 p. 100). Trois genres ont paru relativement représentés : Indigofera (7 espèces), Eragrostis (4 espèces) et Tephrosia (3 espèces). Ce sont aussi des genres qui renferment des espèces de bonne valeur pastorale.

Dans les parcours, la diversité spécifique des différentes catégories fourragères a été assez variable : 14 espèces pour BVP, 21 pour MVP, 22 pour FVP et 3 pour SVP. Les espèces SVP ont été globalement très rares ; il s'agissait de Sida urens, Acalypha segetalis et Mitracarpus scaber. Ces espèces n'ont pas de valeur parce qu'elles ne sont pas consommées !

Le nombre d'espèces dans les différentes catégories a varié aussi selon le temps d'abandon cultural des parcelles (figure 2). Dans les trois premières années de jachère (J1 à $\mathrm{J} 3$ ou jeunes jachères), la distribution des espèces dans les catégories BVP, MVP et FVP est apparue relativement homogène (25 à 35 p. 100). A partir de la cinquième année (J5), les espèces FVP ont été largement dominantes (41 p. 100 dans J5, 47 p. 100 dans J10 et 40 p. 100 dans J17). Dans J10, les espèces BVP ont été particulièrement rares (17 p. 100).

Dans le cortège, ont été identifiées des espèces communes aux différents âges de jachères ou des espèces relativement stables ; il s'agissait d'Eragrostis tremula, de Tephrosia pedicellata Bak. qui tendait à disparaître de la jachère de 17 ans, de Tetraneuris linearifolia, d'Euphorbia macrophylla, de Hyptis spicigera, de Cassia absus et de Walteria indica. Zornia glochidiata et Indigofera pilosa, légumineuses d'excellente qualité fourragère, présentes dans les jachères jeunes (entre 1 et 3 ans), n'ont pas été observées dans les jachères âgées. Andropogon pseudapricus a été absente dans les jachères d'âge intermédiaire ( 5 et 10 ans). Elionurus elegans et Hackelochloa granularis ont été absentes dans la jachère de 17 ans. D'autres espèces ont caractérisé les âges intermédiaires ; il s'agissait de Ceratotheca sesamoides et de Sesbania pachycarpa (3 et 5 ans). D'autres espèces enfin sont apparues avec l'âge de la jachère : Hyptis suaveolens, Ipomoea argentaurata (5 à 17 ans) ou Indigofera hirsuta et Achyranthes argentea (10 à 17 ans).

Les espèces pérennes ont été peu représentées. Dans le groupe des espèces à BVP, les auteurs ont repéré Tephrosia pedicellata qui tendait à disparaître avec l'allongement du temps d'abandon cultural. Sa contribution est apparue ainsi faible, voire insignifiante dans les jachères âgées (recouvrement égal à 0,6 p. 100 dans J17). Dans la catégorie des espèces à MVP, Schizachyrium nodulosum a colonisé $\mathrm{J} 17$, tandis que Indigofera secundiflora et Euphorbia macrophylla ont été présentes respectivement dans $\mathrm{J} 1$ et $\mathrm{J} 5$, donc dans les jeunes jachères. Dans J3, les espèces pérennes rencontrées ont présenté de très faibles recouvrements $(<1$ p. 100$)$; leur contribution est ainsi devenue insignifiante dans la valeur pastorale. 


\section{Tableau I}

Composition de la végétation pastorale et recouvrement moyen des espèces des parcelles de jachères soudaniennes de Saré Yéro Bana, Haute Casamance, sud du Sénégal

\begin{tabular}{|c|c|c|c|c|c|c|c|c|}
\hline Genre et espèce & Famille & $I \mathbf{s}_{\mathrm{i}}$ & $J 1$ & J3 & J5 & $\mathrm{J10}$ & $J 17$ & $\operatorname{Rsm}(\%)$ \\
\hline Effectif des relevés & & & 10 & 22 & 12 & 20 & 18 & 82 \\
\hline Alysicarpus ovalifolius & Fabaceae & 3 & & 11,0 & & 10,3 & 10,2 & 10,5 \\
\hline Andropogon pseudapricus & Poaceae & 3 & 20,1 & 13,6 & & & 17,2 & 17,0 \\
\hline Chloris prieurii & Poaceae & 3 & & & & & 0,1 & 0,1 \\
\hline Dactyloctenium aegyptium & Poaceae & 3 & & 0,1 & & & 2,3 & 1,2 \\
\hline Eragrostis ciliaris & Poaceae & 3 & & & & & 0,6 & 0,6 \\
\hline Eragrostis perbella & Poaceae & 3 & & 0,3 & & & & 0,3 \\
\hline Eragrostis tenella & Poaceae & 3 & & 0,7 & & & 3,4 & 2,1 \\
\hline Eragrostis tremula & Poaceae & 3 & 10,1 & 5,9 & 20,2 & 10,7 & 5,3 & 10,4 \\
\hline Indigofera senegalensis & Fabaceae & 3 & & 0,0 & & & & 0,0 \\
\hline Panicum laetum & Poaceae & 3 & 0,1 & & & & 5,4 & 2,8 \\
\hline Rhynchosia minima & Fabaceae & 3 & & & & 0,1 & & 0,1 \\
\hline Tephrosia bracteolate & Fabaceae & 3 & 0,1 & & 10,0 & & 0,6 & 3,6 \\
\hline Tephrosia linearifolia & Fabaceae & 3 & 0,1 & 1,0 & 1,8 & 2,9 & 0,8 & 1,3 \\
\hline Tephrosia pedicellata & Fabaceae & 3 & 20,1 & 16,7 & 14,0 & 13,8 & 0,6 & 11,0 \\
\hline Zornia glochidiata & Fabaceae & 3 & 0,7 & 0,1 & 11,0 & & & 4,0 \\
\hline Corchorus fascicularis & Tiliaceae & 2 & & & & & 0,1 & 0,1 \\
\hline Cyperus cylindratus & Cyperaceae & 2 & & 0,1 & & 0,1 & & 0,1 \\
\hline Cyperus rotondus & Cyperaceae & 2 & & & & & 4,8 & 4,8 \\
\hline Indigofera pilosa & Fabaceae & 2 & 3,0 & 0,9 & & & & 2,0 \\
\hline Elionurus elegans & Poaceae & 2 & 15,0 & 10,8 & 20,0 & 22,8 & & 17,1 \\
\hline Hackelochloa granularis & Poaceae & 2 & 10,7 & 12,9 & 12,0 & 10,3 & & 11,5 \\
\hline Fimbristylis ferruginea & Cyperaceae & 2 & 1,6 & 0,5 & & 0,3 & & 0,8 \\
\hline Indigofera secundiflora & Fabaceae & 2 & 0,7 & & 4,0 & & & 2,4 \\
\hline Indigofera aspera & Fabaceae & 2 & 0,3 & 14,5 & & 10,6 & 11,4 & 9,2 \\
\hline Setaria pallide-fusca & Poaceae & 2 & 0,3 & & & 0,1 & 0,6 & 0,3 \\
\hline Dolichos stenophylla & Fabaceae & 2 & & 0,5 & & & & 0,5 \\
\hline Indigofera dendroides & Fabaceae & 2 & & 0,3 & & & 0,4 & 0,4 \\
\hline Indigofera hirsute & Fabaceae & 2 & & & & 1,9 & 0,6 & 1,2 \\
\hline Indigofera mucronata & Fabaceae & 2 & & 0,5 & & & & 0,5 \\
\hline Schizachyrium nodulosum & Poaceae & 2 & & & & & 4,9 & 4,9 \\
\hline Achyranthes argentea & Amaranthaceae & 1 & & & & 0,1 & 0,9 & 0,5 \\
\hline Blepharis maderaspatana & Asteraceae & 1 & & & & 0,1 & 0,3 & 0,2 \\
\hline Cassia absus & Caesalpiniaceae & 1 & 3,0 & 1,7 & 8,6 & 6,1 & 1,2 & 4,1 \\
\hline Cassia nigricans & Caesalpiniaceae & 1 & 1,4 & 1,5 & & 0,6 & 0,1 & 0,9 \\
\hline Cassia obtusifolia & Caesalpiniaceae & 1 & & 0,1 & & 0,5 & & 0,3 \\
\hline Ceratotheca sesamoides & Pedaliaceae & 1 & & 0,1 & & & & 0,1 \\
\hline Cissus vogelii & Convolvulaceae & 1 & & & & 0,1 & & 0,1 \\
\hline Desmodium velutinum & Fabaceae & 1 & & & & 0,1 & & 0,1 \\
\hline Euphorbia glomifera & Euphorbiaceae & 1 & & & & & 0,1 & 0,1 \\
\hline Euphorbia macrophylla & Euphorbiaceae & 1 & 2,1 & 0,5 & 3,0 & 1,8 & 10,1 & 3,5 \\
\hline Hibiscus aspera & Malvaceae & 1 & & 0,6 & & & 0,3 & 0,5 \\
\hline Hibiscus diphylla & Malvaceae & 1 & 0,1 & 0,4 & 2,0 & 7,5 & 3,2 & 2,6 \\
\hline Hyptis spicigera & Amaranthaceae & 1 & 22,9 & 21,1 & 14,0 & 21,0 & 17,5 & 19,3 \\
\hline Merremia aegyptiaca & Convolvulaceae & 1 & & 0,2 & & & & 0,2 \\
\hline Merremia pinnata & Convolvulaceae & 1 & & & & & 0,5 & 0,5 \\
\hline Sesbania pachycarpa & Fabaceae & 1 & & 1,4 & 2,0 & & & 1,7 \\
\hline Acalypha segetalis & Euphorbiaceae & 0 & & 0,5 & & & & 0,5 \\
\hline Hyptis suaveolens & Amaranthaceae & 0 & & & 0,4 & 0,6 & 1,2 & 0,7 \\
\hline Ipomoea argentaurata & Convolvulaceae & 0 & & 0,0 & 0,2 & 0,1 & 0,2 & 0,1 \\
\hline Ipomoea coptica & Convolvulaceae & 0 & & & & 0,1 & & 0,1 \\
\hline Ipomoea eriocarpa & Convolvulaceae & 0 & & 0,1 & & 0,1 & & 0,1 \\
\hline Ipomoea pes-tigridis & Convolvulaceae & 0 & & & & & 0,7 & 0,7 \\
\hline Mitracarpus scaber & Rubiaceae & 0 & & 0,0 & & & 0,2 & 0,1 \\
\hline Sida urens & Malvaceae & 0 & & 1,2 & & & & 1,2 \\
\hline Spermacoce chaetocephala & Rubiaceae & 0 & 0,4 & 0,6 & & 0,1 & 4,7 & 1,4 \\
\hline
\end{tabular}




\section{Tableau I (suite)}

Composition de la végétation pastorale et recouvrement moyen des espèces des parcelles de jachères soudaniennes de Saré Yéro Bana, Haute Casamance, sud du Sénégal

\begin{tabular}{|c|c|c|c|c|c|c|c|c|}
\hline Genre et espèce & Famille & $\mathbf{I s}_{\mathbf{i}}$ & J1 & J3 & $\mathbf{J 5}$ & $J 10$ & $J 17$ & $\operatorname{Rsm}(\%)$ \\
\hline Spermacoce radiata & Rubiaceae & 0 & & 0,0 & & & & 0,0 \\
\hline Spermacoce stachydea & Rubiaceae & 0 & & 3,3 & 0,4 & 0,2 & 6,5 & 2,6 \\
\hline Stylochiton warneckiii & Araceae & 0 & & & & 0,1 & & 0,1 \\
\hline Waltheria indica & Sterculiaceae & 0 & 0,9 & 0,6 & 1,2 & 1,8 & 10,0 & 2,9 \\
\hline Richesse floristique & & 60 & 21 & 39 & 17 & 30 & 35 & \\
\hline
\end{tabular}

Is $_{\mathrm{i}}:$ indice spécifique

$\mathrm{J} 1$ : jachère de 1 an ... $\mathrm{J} 17$ : jachère de 17 ans

Rsm : recouvrement spécifique moyen

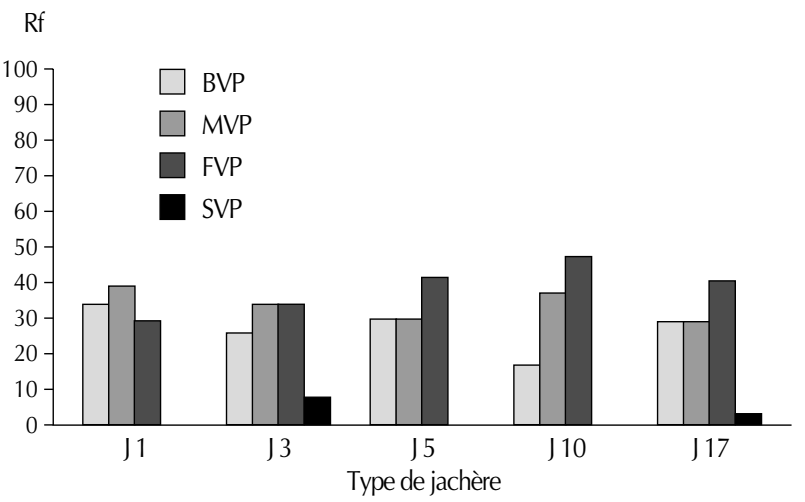

Figure 2 : diversité des différentes catégories d'espèces fourragères (Rf) dans les différentes parcelles de jachère. Plantes: $B V P=$ de bonne valeur pastorale $; M V P=$ de moyenne valeur pastorale ; $F V P=$ de faible valeur pastorale $; S V P=$ sans valeur pastorale.

\section{Indice global de qualité des herbages de jachères soudaniennes}

Le recouvrement spécifique moyen (Rsm) (tableau I) est apparu faible : pour l'ensemble des jachères étudiées, peu d'espèces ont présenté un recouvrement moyen supérieur ou égal à 10 p. 100 ; il s'agissait de Hyptis spicigera Lam. (19,3 p. 100), Elionurus elegans Kunth. (17,1 p. 100), Andropogon pseudapricus Stapf. (17 p. 100), Hackelochloa granularis O. Kze (11,5 p. 100), Tephrosia pedicellata (11 p. 100), Alysicarpus ovalifolius (Schumach. et Thonn.) Léonard (10,5 p. 100) et Eragrostis tremula Hochst. (10 p. 100). Dans ce lot, deux espèces seulement, $T$. pedicellata et $A$. ovalifolius, ont présenté un indice de qualité égal à 3 ; ce sont des espèces de bonne valeur pastorale. E. elegans est une espèce à moyenne valeur pastorale, tandis que $H$. spicigera (l'espèce la plus abondante) est une plante sans valeur pastorale. Quatre autres espèces, Indigofera aspera Perr. ex DC. (6,7 p. 100), Cassia obtusifolia L. (6,1 p. 100), Cyperus sp. et Schizachyrium brevifolium (Sw.) Nees (5 p. 100) ont pu être considérées comme moyennement productives avec un recouvrement de l'ordre de 5 p. 100.

Les valeurs pastorales relatives des espèces $\left(\mathrm{Vr}=\mathrm{Cs}_{\mathrm{i}} \mathrm{x} \mathrm{Is}_{\mathrm{i}}\right)$ ont été peu élevées. A. pseudapricus $(35,4)$, E. elegans $(23,8)$, T. pedicellata (23), E. tremula $(21,8)$, A. ovalifolius $(21,8), H$. granularis $(15,9)$, H. spicigera $(13,4)$, Zornia glochidiata Reichb. ex DC.
$(8,2)$ et Tephrosia bracteolata Guill. et Perr. $(7,5)$ ont présenté les valeurs les plus élevées dans les parcelles de jachère. H. spicigera, qui a présenté la contribution spécifique la plus élevée de cette étude (19,3 p. 100), n'est arrivée qu'au sixième rang ; c'est en effet une espèce de faible valeur pastorale $\left(\mathrm{Is}_{\mathrm{i}}=1\right)$.

Dans l'indice global de qualité des parcours de jachères, les catégories d'espèces fourragères BVP et FVP ont présenté des valeurs pastorales relatives similaires, soit 54,7 (tableau II). Elles ont été plus élevées $(35,7)$ que celles de MVP. La valeur des parcours de jachères en Haute Casamance a été de 48,3 \pm 2,7 (tableau II). Les contributions des catégories d'espèces dites BVP et FVP ont été équivalentes : 37,7 p. 100 (tableau III) ; elles ont été les plus importantes tandis que celles de MVP n'ont représenté que 24,6 p. 100 de la valeur de l'indice.

\section{Variation de la valeur pastorale en fonction de la durée d'abandon cultural}

La richesse spécifique de la flore pastorale a varié selon l'âge de la jachère. Le nombre d'espèces fourragères inventoriées a été de 21 dans la parcelle J1, 39 dans J3, 17 dans J5, 30 dans J10 et 35 dans J17. Elle a été assez variable au cours des cinq premières années, puis elle s'est stabilisée dans les jachères âgées (entre 30 et 35 espèces dans $\mathrm{J} 10$ à $\mathrm{J} 17$ ). La richesse spécifique a aussi varié dans les catégories fourragères (figure 3). La répartition de l'effectif des espèces dans les différentes catégories a été assez régulière dans les trois premières années. Dans les jachères âgées, en revanche, est apparue une dominance particulière des plantes FVP au détriment de BVP.

Les contributions spécifiques au spectre fourrager ont montré que les plantes de faible valeur pastorale ont été partout plus importantes que les autres catégories. Elles ont participé en effet pour 40 (J17) à 81 p. 100 (J10) de l'indice global de qualité. Dans la parcelle de 17 ans, les contributions des plantes BVP, MVP et SVP ont été sensiblement équivalentes, soit respectivement 17,2, 19,3 et 23,4 p. 100. La catégorie de plantes SVP n'a pas été représentée dans les classes d'âges inférieures (1, 3, 5 et 10 ans). Les valeurs relatives des différentes catégories fourragères ont varié de 42,5 à 84,6 p. 100 pour les BVP, de 9,2 à 38,6 p. 100 pour les MVP et de 40,1 à 80,9 p. 100 pour les FVP. Elles ont été plus élevées pour les plantes de bonne valeur dans les jeunes jachères. La jachère de 10 ans a renfermé plus d'espèces de faible valeur pastorale que toutes les autres.

La valeur pastorale des parcours de jachères étudiés a varié de 43,4 à 56,3 (figure 3). Elle a sensiblement augmenté dans les trois 


\section{Tableau II}

Variation des espèces dominantes dans les différents types de jachère et valeur pastorale globale

\begin{tabular}{|c|c|c|c|c|c|}
\hline Catégories & $\mathbf{I s}_{\mathbf{i}}$ & $\mathrm{Cs}_{\mathrm{i}}$ & $\mathrm{Vr}$ * & $\mathbf{R f}$ & Espèces dominantes \\
\hline BVP & 3 & 18,2 & 54,7 & 14 & $\begin{array}{l}\text { Tephrosia pedicellata, Eragrostis tremula, Andropogon pseudapricus, } \\
\text { Alysicarpus ovalifolius }\end{array}$ \\
\hline MVP & 2 & 17,9 & 35,7 & 21 & $\begin{array}{l}\text { Hackelochloa granularis, Elionurus elegans, Indigofera aspera, } \\
\text { Schizachyrium brevifolium, Cyperus sp. }\end{array}$ \\
\hline FVP & 1 & 54,7 & 54,7 & 22 & Hyptis spicigera, Cassia obtusifolia, Spermacoce sp., Walteria indica \\
\hline SVP & 0 & 9,3 & 0,0 & 4 & Sida urens, Mitracarpus scaber \\
\hline
\end{tabular}

$\mathrm{Is}_{\mathrm{i}}$ : indice de qualité $; \mathrm{Cs}_{\mathrm{i}}$ : contribution spécifique $; \mathrm{Vr}$ : valeurs pastorales relatives des espèces

$* \mathrm{Vr}=\mathrm{Cs}$. $\mathrm{X} I \mathrm{~s}$

Rf : richesse spécifique

BVP : plantes de bonne valeur pastorale ; MVP : plantes de moyenne valeur pastorale ; FVP : plantes de faible valeur pastorale ; SVP : plantes sans valeur pastorale

\section{Tableau III}

Paramètres d'évaluation de fourrage qualifié et charge animale des parcours de jachères soudaniennes de Saré Yéro Bana en Haute Casamance, sud du Sénégal

\begin{tabular}{lccccc} 
Parcelles de jachères & $\mathbf{J 1}$ & $\mathbf{J 3}$ & $\mathbf{J 5}$ & $\mathbf{J 1 0}$ & $\mathbf{J 1 7}$ \\
\hline Production (kg MS*/ha) & 2250 & 2400 & 3250 & 4150 & 4600 \\
Valeur pastorale brute & 50,3 & 56,4 & 46,3 & 44,4 & 43,4 \\
Recouvrement global (\%) & 90,0 & 90,0 & 80,0 & 65,0 & 60,0 \\
Valeur pastorale nette & 45,3 & 50,7 & 37,0 & 28,9 & 26,0 \\
Fourrage qualifié (kg MS/ha) & 1019 & 1217 & 1202 & 1200 & 1196 \\
Capacité de charge (ubt**/ha) & 0,91 & 0,93 & 0,92 & 0,91 & 0,91
\end{tabular}

$\mathrm{J} 1$ : jachère de 1 an ... $\mathrm{J} 17$ : jachère de 17 ans

* Matière sèche

** Unité de bétail tropical

premières années de 50,3 à 56,3, puis a diminué entre 5 et 17 ans de 46,3 à 43,4. La contribution des différentes catégories n'a pas semblé liée à la richesse spécifique. Dans J3, par exemple, l'effectif des espèces dans les différentes catégories a été équivalent, alors que seules les plantes BVP et FVP ont contribué de manière significative. La parcelle J5, dont la richesse spécifique a été la plus faible, a

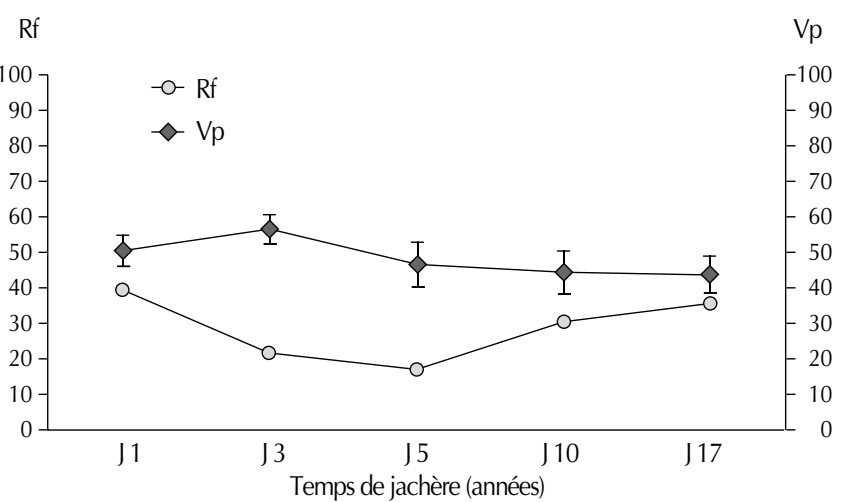

Figure 3 : variations de la valeur pastorale (VP) et de la richesse spécifique $(R f)$ des parcours de jachères soudaniens selon le temps d'abandon cultural. présenté une valeur pastorale nettement plus élevée que celle des jachères âgées. Dans les jachères âgées, le nombre d'espèces a été le plus élevé dans la catégorie FVP, tandis que la contribution des catégories fourragères à l'indice global a paru très équitable.

Ces résultats ont indiqué une baisse de la qualité fourragère de ces herbages depuis les jeunes jachères jusqu'à la savane reconstituée (J17). Cette baisse a été encore plus accentuée avec la valeur pastorale nette (tableau III). La valeur pastorale nette a été obtenue en pondérant la valeur pastorale par le recouvrement global ou couvert herbacé. Le couvert herbacé a varié de 60 (J17) à 90 p. 100 (J1 et J3). Il a été assez élevé dans les jeunes jachères, comparé à celui observé dans les jachères âgées. L'indice global de qualité et de richesse spécifique déterminé pour ces milieux de jachères soudaniennes a évolué relativement peu dans les parcelles étudiées.

\section{Fourrage qualifié produit et charge animale}

Appliquée à la phytomasse herbacée épigée (tableau III), la valeur pastorale nette (VPn) a permis de qualifier le fourrage produit (11). Ainsi, pour les parcelles de jachère âgée (J10 et J17), la production de 4150 et $4600 \mathrm{~kg} / \mathrm{ha} / \mathrm{an}$ de matière sèche, avec respectivement une VP de 44 et 43, n'équivaut qu'à 1200 et $1196 \mathrm{~kg} / \mathrm{ha} / \mathrm{an}$ de fourrage qualifié. En revanche, dans les jachères jeunes (J1 et J3), les productions de phytomasse de 2250 et $2400 \mathrm{~kg} / \mathrm{ha} / \mathrm{an}$ de 
matière sèche, avec une VP de 50 et 56 , étaient équivalentes à 1019 et $1217 \mathrm{~kg} / \mathrm{ha} / \mathrm{an}$ de fourrage qualifié.

La capacité de charge correspond au nombre de têtes de bétail qu'un parcours peut accueillir et nourrir sans se détériorer. Elle a varié de 0,91 à $0,93 \mathrm{ubt}^{1} / \mathrm{ha}$. La charge de ces milieux a paru presque entièrement stable. Cette pondération de fourrage qualifié améliore l'estimation de la charge possible en bétail dans les jeunes jachères, car la part de fourrage inutilisée peut être réduite de 50 p. 100 (pertes biologiques au cours de la saison sèche, pertes par les prédateurs, pertes par piétinement...).

\section{DISCUSSION ET CONCLUSION}

On détermine généralement la valeur alimentaire du fourrage selon deux approches : une approche phytoécologique et une approche zootechnique ou analytique. Pour qualifier le fourrage des parcours de jachères du terroir de Saré Yéro Bana en Haute Casamance, au sud du Sénégal, l'approche phytoécologique qui repose sur la détermination de la valeur pastorale a été utilisée. Les auteurs ont utilisé la valeur pastorale comme une note pour comparer les différents âges des jachères et suivre leur évolution dans le temps pour une utilisation efficace de la végétation de ces milieux. Des observations ont été menées sur des sites pour lesquels aucun contrôle des facteurs du milieu n'avait été a priori réalisé. Elles ont alors consisté en des relevés de végétation dans des parcelles de jachère d'âges différents.

La détermination de la valeur pastorale combine la composition, la contribution et l'indice de qualité des espèces herbacées. L'indice de qualité traduit l'intérêt zootechnique de chaque espèce herbacée pour une ou plusieurs espèces animales (9) et a concerné, dans le cadre de cette étude, les bovins, les ovins et les caprins. L'intérêt zootechnique recouvre la palatabilité, la productivité et la valeur alimentaire de l'espèce. Une telle approche présente sans nul doute des forces et des faiblesses.

\section{Faiblesses de la méthode utilisée}

Les indices spécifiques sont constants dans le temps et dans l'espace (sahélien, par exemple), alors que les propriétés nutritives d'une espèce varient selon les saisons et les écotypes. De même, la contribution spécifique tient compte aussi bien des espèces fourragères que des espèces non fourragères. Ainsi, si on ajoute des espèces non fourragères dans un parcours composé uniquement d'espèces fourragères, la valeur pastorale baisse. Des auteurs (15) se sont demandés comment cette baisse se justifiait alors que les espèces non fourragères n'entravaient pas la consommation des espèces fourragères.

La composante animale n'est pas non plus prise en compte dans le calcul de la valeur pastorale. En effet, le type d'animal et les modalités de pâturage ne sont pas spécialement intégrés dans le mode d'évaluation. Il en est de même pour le comportement alimentaire, car les animaux choisissent ce qu'ils mangent et la végétation ingérée est parfois différente de la végétation sur pied. Parce que l'animal choisit sa ration, un parcours de faible valeur pastorale n'est pas forcément de mauvaise qualité et la ration ingérée peut s'avérer excellente. La sélection s'opère à trois niveaux : les organes végétaux, les espèces et les zones de pâturages $(17,18)$. La méthode s'appuie sur les espèces et les zones de pâturages pour déterminer la qualité des parcours. Elle n'aborde donc pas les organes végétaux.

\footnotetext{
${ }^{1}$ Unité de bétail tropical, soit un animal de $250 \mathrm{~kg}$ de poids vif et consommant $6,25 \mathrm{~kg}$ de matière sèche par jour
}

A propos de ce tri par les animaux, Pechanec et Pickford (24) ont fait remarquer qu'une méthode d'évaluation, pour être efficace, doit répondre à trois critères fondamentaux : la rapidité, la précision et la souplesse. Or, une des raisons de la notion de valeur pastorale est sa simplicité d'utilisation. Si cet indice gagne en précision, il perd en rapidité d'exécution et de souplesse.

Enfin, la méthode utilisée ne considère que le tapis herbacé et ne prend pas en compte les ligneux. En effet, la valeur pastorale n'est opérationnelle que dans les parcours où l'essentiel de leurs ressources est sous forme herbacée (15). C'est le cas des pâturages de jachères de la zone étudiée. Dans les parcours où la strate arbustive occupe une place importante de la ressource, l'étude de la valeur pastorale, basée sur les espèces herbacées, parait ainsi ne plus suffire pour établir la qualité de la ressource disponible. Malgré ces limites, l'approche permet de montrer l'existence de relations entre la diversité des espèces, le temps d'abandon cultural, le moment d'utilisation et la valeur des parcours de jachères de la Haute Casamance.

\section{Diversité spécifique et valeur des parcours de jachères}

La valeur pastorale dépend en premier lieu des espèces présentes dont l'appétabilité conditionne la fréquentation et l'ingestion. Certaines espèces en effet sont recherchées par les animaux du troupeau (espèces très appétées), d'autres sont consommées à l'occasion sans que les animaux les recherchent, d'autres enfin sont délaissées (espèces non appétées), parfois même en cas de famine (9). Parmi ces espèces, les unes sont particulièrement intéressantes par leur production (espèces productrices), par leur qualité (espèces de bonne valeur fourragère), d'autres le sont moins. Les Peuhls, qui suivent avec attention le comportement de leur troupeau, savent que certaines herbes, certaines plantes « font du lait, du beurre et/ou de la viande ». Le concept d'indice spécifique de qualité traduit ce classement de l'espèce. Cet indice tient compte de la vitesse de croissance de l'espèce, de sa palatabilité, de son assimilabilité et de sa résistance à la dent. L'indice permet ainsi de situer les espèces les unes par rapport aux autres, des pires (espèces sans valeur pastorale) aux meilleures (espèces dites de bonne valeur pastorale). L'indice nul (espèce sans valeur pastorale) ne signifie pourtant pas que l'animal ne consomme pas la plante, mais plutôt que celle-ci lui est peu profitable. Cette plante joue pourtant un rôle essentiel dans l'amélioration du coefficient d'encombrement.

La valeur pastorale varie aussi avec l'importance (ou abondance relative) des espèces présentes, c'est-à-dire leur contribution $\left(\mathrm{Cs}_{\mathrm{i}}\right.$, calculée ici sur la base du recouvrement des espèces) au spectre fourrager. Dans les parcelles de jachères étudiées, une partie importante de la flore herbacée a été représentée par des espèces productives, c'est-à-dire présentant des contributions supérieures ou égales à 1 p. 100 (10). Peu d'entre elles, cependant, ont été de bonne valeur pastorale ; cela a réduit les valeurs relatives pastorales spécifiques et, par conséquent, l'indice global de qualité des parcours.

Par ailleurs, la valeur pastorale est pondérée par le recouvrement ; cela permet de s'affranchir du problème de surestimation. Ainsi, une bonne végétation, caractérisée par une valeur pastorale de 70 , donnera au pâturage une valeur de 7, s'il y a 90 p. 100 de sol nu, et 35 s'il y a 50 p. 100 de sol nu.

\section{Temps d'abandon cultural et valeur des parcours de jachères}

Enfin, dans les parcours de jachères de Saré Yéro Bana, l'indice global de qualité des pâturages herbacés a varié avec la durée d'abandon cultural. La valeur pastorale a été relativement faible 
dans les jachères âgées (10 et 17 ans). Dans ces terres de parcours de jachères âgées, le couvert ligneux est aussi devenu plus important ; cela a réduit considérablement le tapis herbacé. L'indice global de qualité dans la parcelle de jachère de 3 ans a été le plus élevé. Cette jachère s'est caractérisée par la richesse spécifique la plus importante, un nombre d'espèces productives (6 sur 15) plus important (E. tremula, Z. glochidiata, E. elegans, I. aspera, $H$. granularis et $H$. spicigera). Dans ce parcours, la végétation ligneuse a été représentée par les Combretaceae (Terminalia sp. et Combretum sp.). Dans certaines zones, des espèces ligneuses des jachères ont été fourragères ; elles ont contribué à l'alimentation du bétail.

Dans les jeunes jachères (1 à 3 ans), la valeur pastorale a été relativement élevée. Toute la matière sèche produite a été entièrement consommée par le bétail entre novembre et janvier ou février (1). Tout s'est passé comme dans les formations sahéliennes à espèces annuelles. Les plantes annuelles constituent en effet l'essentiel du spectre fourrager. Kiéma (22) et Ouédraogo (23) ont aussi montré au Burkina Faso que les plantes annuelles des jachères sont des espèces fourragères de très bonne qualité.

Les jachères anciennes ont donc été pauvres dès le début de la saison sèche, car leur tapis herbacé a été essentiellement constitué de grandes graminées : Andropogon pseudapricus et Schizachyrium sp., par exemple. Malgré une forte production, la capacité de charge de ces parcours n'a pas été supérieure à celle des jeunes jachères $(0,91 \mathrm{ubt} / \mathrm{ha})$. Elles deviennent toutefois utiles dans la pleine saison sèche, car après le passage des feux de brousse, les plantes herbacées pérennes rejettent pour remplacer les pâturages herbacés (d'espèces annuelles) déjà épuisés. César et Zoumana (8), étudiant la végétation de deux parcelles de jachère, l'une récente, l'autre ancienne, soumises à une pâture intense montrent que la valeur pastorale d'une jachère dépend beaucoup plus de l'intensité d'utilisation que de son âge ou de son stade d'évolution vers la savane. Ils constatent en fait que les valeurs pastorales de ces deux milieux sont similaires malgré une proportion beaucoup plus importante de graminées vivaces dans la jachère ancienne.

\section{Moment d'utilisation et valeur des parcours de jachères}

Les parcours de jachères sont ouverts au bétail toute l'année, tout au moins pour le troupeau local. Ils sont donc parcourus continuellement. Toutefois, du fait des activités champêtres et de l'accessibilité de ces parcours, la période d'utilisation optimale se limite à la saison des pluies pour les jachères âgées. Les jeunes jachères servent généralement de remparts pour protéger les cultures. Entre novembre et janvier, les parcours de jachères sont peu fréquentés car les adventices et les résidus de culture sont directement pâturés sur les champs. En milieu et en fin de saison sèche, les animaux reviennent sur les parcelles de jachère pour y exploiter les feuilles, ou fruits de ligneux et la paille.

Dans des conditions d'élevage extensif, les parcelles de jachère représentent une source importante de fourrages. L'indice global de qualité peut constituer un indicateur essentiel de valeur fourragère de ces parcours en l'absence d'analyses bromatologiques, généralement longues et onéreuses. La contribution des jachères à l'alimentation du bétail est d'autant plus importante qu'elle assure, en saison des pluies, une grande partie des productions animales annuelles. L'utilisation des pâturages de jachères par le bétail devrait donc permettre de diversifier les sources d'alimentation du bétail et, ainsi, de réduire la pression qui s'exerce sur les zones de parcours naturelles. En effet, la jachère en tant qu'espace pastoral prendra toujours plus de valeur au fur et à mesure que les parcours naturels seront réduits pour laisser place aux cultures (6).
Dans le cadre des programmes de gestion intégrée des terroirs (utilisation pastorale des parcelles de jachère), une période de trois ans d'abandon cultural semble suffisante pour que l'animal tire un profit maximal de la végétation herbacée des parcelles de jachère. Dans ces milieux, l'animal contribue aussi à améliorer la fertilité des sols par ses déjections (fèces, urines) ; cela peut considérablement réduire le temps de jachère nécessaire à la remontée (naturelle) de la fertilité des sols. Au-delà, l'équilibre entre les différentes catégories d'espèces fourragères est rompu et les plantes FVP deviennent généralement plus abondantes. Les milieux de jachères, bien que très modifiés par les activités humaines, sont indubitablement assez productifs, au sens écologique du terme. Ils représentent une source alimentaire particulièrement intéressante pour le bétail. Toutefois, il semble important de poursuivre les recherches pour comparer la valeur pastorale et la capacité de charge des jachères et des parcours naturels du sud du Sénégal.

Les quantités en éléments nutritifs et la digestibilité du matériel végétal produit déterminent la valeur alimentaire (7), mais la valeur pastorale représente un élément d'appréciation important dans l'évaluation des terres de parcours. L'utilisation de la phytomasse herbacée pour la production secondaire se heurte presque toujours, en effet, au problème de la qualité fourragère.

\section{BIBLIOGRAPHIE}

1. AKPO L.E., GROUZIS M., 2000. Valeur pastorale des herbages en région soudanienne : le cas des parcours sahéliens du Nord-Sénégal. Tropicultura, $18: 1-8$.

2. BANOIN M., ACHARD F., 1998. Place des jachères dans les systèmes d'élevage et comportement alimentaire du bétail sur un terroir du sudouest (Ticko) du Niger. In : Floret C., Pontanier R. eds, Jachère et systèmes de production en Afrique de l'Ouest. Paris, France, Orstom, p. 99-110.

3. BARRAL H., BENEFICE E., BOUDET G., DENIS J.F., DE WISPELAERE G., DIAITE I., DIAW O.T., DIEYE K., DOUTRE M.P., MEYER J.F., NOEL J., PARENT O., PIOT J., PLANCHENAULT D., SANTOIR C., VALENTIN C., VALENZA J., VASSILIADES G., 1983. Systèmes de production d'élevage au Sénégal dans la région du Ferlo. ACC/Grizat (LAT). Paris, France, Isra/Orstom, $172 \mathrm{p}$.

4. BERHAUT J., 1967. Flore du Sénégal. Dakar, Sénégal, Clairafrique, $445 \mathrm{p}$.

5. BLANFORT V., 1991. Phytosociologie et production de la végétation des parcours d'une petite région agropastorale de Moyenne Casamance (Sénégal). Analyse de la variabilité pour une application à l'étude des systèmes d'alimentation des ruminants. Maisons-Alfort, France, Cirademvt, $127 \mathrm{p}$

6. BONETTI $C$. JOUVE $P$, 1991. Jachères et systèmes agraires en Afrique subsaharienne : synthèse bibliographioque. In : Amélioration et gestion de la jachère en Afrique de I'Ouest. Dakar, Sénégal, Coraf/UE/IRD.

7. BREMAN H., DE RIDDER N., 1991. Manuel sur les pâturages des pays sahéliens. Paris, France, Acct/Karthala, $485 \mathrm{p}$.

8. CESAR J., ZOUMANA C., 1991. Conséquence de I'accroissement démographique sur la qualité de la jachère dans le nord de la Côte d'Ivoire. In : Floret C., Serpantié G. éds, La jachère en Afrique de I'Ouest. Paris, France, Orstom, p. 415-434. (Colloques et séminaires)

9. DAGET P., GODRON M., 1995. Pastoralisme : troupeaux, espaces et sociétés. Paris, France, Hatier/Aupelf.

10. DAGET P., POISSONET J., 1971. Une méthode d'analyse phytologique des prairies. Critères d'application. Ann. Agron., 22 : 5-41.

11. DAGET P., POISSONET J., 1991. Notion de valeur pastorale. Repères, 2 : 4-8.

12. FANCHETTE S., 1994. Gestion d'un espace pastoral en expansion, le Fuladu (région de Kolda, Sénégal). Rapport de stage. Paris, France, Orstom, 8 p. 
13. FELLER C., LAVELLE P., ALBRECHT A., NICOLARDOT B., 1991. La jachère et le fonctionnement des sols tropicaux : rôle de l'activité biologique et des matières organiques. Quelques éléments de réflexion. In : Floret C., Serpantié G. éds, La jachère en Afrique de l'Ouest. Paris, France, Orstom, p. 16-32. (Colloques et séminaires)

14. FLORET C., SERPANTIE G. éds, 1991. La jachère en Afrique de I'Ouest. Paris, France, Orstom, 494 p. (Colloques et séminaires)

15. GARDE L., SENN O., 1991. Valeur pastorale et parcours méditerranéens. In : Gaston et coll. éds, actes $4^{\mathrm{e}}$ Congrès international des terres de parcours, Montpellier, France, avril 1991, p. 458-461.

16. HIERNAUX P., FERNANDEZ-RIVERA S., SCHLECHT E., TURNER M.D. WILLIAMS T.O., 1998. Livestock-mediated nutrient transfers in Sahelian ecosystems. In: Renard G., Neef A., Becker K., von Oppen M. Eds, Soil fertility management in West African land use systems. Weikersheim, Germany, Margraf Verlag, p. 339-347.

17. HIRCHE A., 1994. Sur la notion de valeur pastorale. Parcours demain ( ${ }^{\circ}$ spécial) : 85-88.

18. ICKOWICZ A., USENGUMUREMYI J., BADIANE A., RICHARD D., COLLEYE F., DUPRESSOIR D., 1998. Interactions entre jachère et systèmes d'alimentation des bovins. Choix technique et dynamique de développement (zone soudanienne, Sénégal). In : Floret, Pontanier éds, La jachère et les systèmes de production en Afrique de I'Ouest. Paris, France, Orstom, p. 123-138

19. JOUVE P.M., 1991. Usages et fonctions de la jachère en Afrique de I'Ouest et au Maghreb. In : Floret C., Serpantié G. éds, La jachère en Afrique de I'Ouest. Paris, France, Orstom, p. 55-66. (Colloques et séminaires)

20. KAREMBE M., YOSSI H., DIALLO M., COULIBALY M., 1998. Jachères et système d'élevage en zone soudanienne du Mali (terroir villageois de Missira). In : Floret, Pontanier éds, La jachère et les systèmes de production en Afrique de l'Ouest. Paris, France, Orstom, p. 100-122.
21. LEBRUN J.P., STORK A., 1991, 1992, 1995. Enumération des plantes à fleurs d'Afrique tropicale, 4 volumes. Genève, Suisse, Conservatoire et Jardin botaniques.

22. KIEMA S., 1992. Utilisation pastorale des jachères dans la région de Bondoukuy, zone soudanienne du Burkina Faso. Mémoire DESS, Gestion des systèmes agrosylopastoraux en zone tropicale, université de Paris XII, France, $89 \mathrm{p}$.

23. OUEDRAOGO M., 1993. Ecologie comparée de deux espèces de graminées pérennes, Andropogon ascinodis C.B. Cl. et Schizachyrium sanguineum (Retz.), dans la région de Bondoukuy (Burkina Faso). Mémoire IDR, université d'Ouagadougou, Burkina Faso, 72 p.

24. PECHANEC J.F., PICKFORD G.D., 1937. A comparison of some methods used in determinating percentage utilisation of range grasses. J. Agric. Res., 54: 753-765.

25. PNUE, 1988. Introduction au projet Ecosystèmes pastoraux sahéliens : rapport général. Gems, Série : Sahel. Rome, Italie, PNUE/ONU-AA, $145 \mathrm{p}$.

26. RICHARD D., AHOKPE B., BLANFORT V., POUYE B., 1991. Utilisation des zones agricoles et pastorales par les ruminants en zone soudanienne (Moyenne Casamance, Sénégal). In : Gaston et coll. éds, actes $4^{\mathrm{e}}$ Congrès international des terres de parcours, Montpellier France, avril 1991, p. 759-762.

27 RIPPSTEIN G., 1991. Une méthode d'étude et de classification des pâturages de savane. In : Gaston et coll. éds, actes $4^{\mathrm{e}}$ Congrès international des terres de parcours, Montpellier, France, avril 1991, p. $1435-1436$.

28 SING J.S., LAUENROTH W.K., STEINHORST R.K., 1975. Review and assessement of various techniques for estimating an aerial primary production in grasslands from harvest data. Bot. Rev., 41: 181-232.

Accepté le 20.11.2003

\section{Summary}

Akpo L.E., Masse D., Grouzis M. Length of Fallow Period and Pastoral Value of Herbaceous Plants in the Sudanese Zone of Senegal

The pasture value of Sudanese fallows was determined for the Sare Yero Bana soil of High Casamance in Senegal by studying fodder diversity and the global quality index. Fodder diversity was higher in young fallow rangelands. In old fallows, on the other hand, species with low pastoral value were relatively more abundant. The global quality index of rangelands markedly increased in fallow first years, peaked the third year because of grass abundance and sparse woody cover, then decreased between years 5 and 17 . The decrease of the fodder quality of fallow rangelands was even more pronounced when estimating the net pastoral value. The cattle carrying capacity of young fallows improved when ponderation of "qualified" fodder was taken into account, because the amount of unused fodder could be reduced by $50 \%$. The global quality index is therefore an essential indicator of rangelands fodder quality, which can be used without the need for chemical and/or bromatological analyses.

Key words: Livestock - Rangeland - Grazing land - Feed crop - Fallow - Quality - Senegal.

\section{Resumen}

Akpo L.E., Masse D., Grouzis M. Duración del barbecho y valor pastoril de la vegetación herbácea en la zona sudanesa

Se determinó el valor pastoril de las parcelas en barbecho sudanesas, en la zona de Saré Yéro Bana, en Alta Casamancia, mediante el estudio el espectro forrajero y el índice global de calidad de las tierras de trayecto. Se observó una mayor diversidad de especies forrajeras en los terrenos en barbecho reciente. En las parcelas más viejas, se observó, por el contrario, una abundancia relativa de especies de bajo valor pastoril. El índice global de la calidad de los terrenos aumentó sensiblemente durante los primeros años de abandono de cultivo, culminando durante el tercer año gracias a la abundancia de gramíneas y a la baja cobertura de leñosas, disminuyendo luego entre 5 y 17 años. Esta disminución en la calidad forrajera de los terrenos en barbecho fue todavía más acentuada cuando se evaluó el valor pastoril neto. La toma en consideración de la ponderación del forraje "calificado", mejoró la carga de ganado de los barbechos recientes, debido a que la porción de forraje no utilizado pudo reducirse a 50\%. En ausencia de cualquier análisis químico o bromatológico, el índice de calidad puede así constituir un indicador esencial de la calidad forrajera de los terrenos.

Palabras clave: Ganado - Tierra de pastos - Tierra de pastoero - Planta forrajera - Barbecho - Calidad - Senegal 